\title{
Efficacy, safety, and applicability of outpatient treatment for diverticulitis
}

\author{
This article was published in the following Dove Press journal: \\ Drug, Healthcare and Patient Safety \\ 31 March 2014 \\ Number of times this article has been viewed
}

\section{Antonio Tursi \\ Gastroenterology Service, ASL BAT, Andria BT, Italy}

Correspondence: Antonio Tursi Gastroenterology Service, ASL BAT, Via Torino 49, 76I23 Andria BT, Italy Tel +3908 83551094

Fax +3908831978210

Email antotursi@tiscali.it
Abstract: Acute diverticulitis of the colon represents a significant burden for national health systems, in terms of direct and indirect costs. Although current guidelines recommend use of antibiotics for the outpatient treatment of acute uncomplicated diverticulitis, evidence for this is still lacking. Hence, significant effort is now being made to identify the appropriate therapeutic approach to treat and prevent relapses of diverticulitis. Outpatient treatment has been identified as a safe and effective therapeutic approach in up to $90 \%$ of patients with uncomplicated diverticulitis. It allows important costs saving to health systems without a negative influence on quality of life for patients with uncomplicated diverticulitis, and reduces health care costs by more than $60 \%$.

Keywords: diverticulitis, 5-aminosalycilic acid, antibiotics, probiotics, outpatient treatment

\section{Introduction}

Colonic diverticulosis is characterized by the presence of pockets that occur when colonic mucosa and submucosa herniate through defects in the muscle layer of the colon wall. ${ }^{1}$ Diverticulosis is commonly found in the western world. Although it has been thought that diverticulosis is a rare condition in developing nations, there is some indication that the prevalence of colonic diverticulosis is increasing throughout the world, probably because of changes in lifestyle. ${ }^{2}$ Although most people with diverticulosis remain asymptomatic, about one quarter of them will develop an episode of symptomatic diverticular disease and up to $5 \%$ an episode of acute diverticulitis. ${ }^{3}$ Acute diverticulitis, defined as an acute inflammation of the colonic diverticula, ${ }^{1}$ shows an increasing prevalence. ${ }^{4}$

Although we currently have much more information about the etiology, disease course, and treatments available to diagnose and manage acute diverticulitis, the treatment options are not well defined because of a lack of evidence. In particular, there are few systematic reviews and well conducted trials to help decision-making in the diagnosis and treatment of diverticulitis, especially on an outpatient basis. This review describes the current management of acute colonic diverticulitis, with a focus on the safety and effectiveness of outpatient treatment of the disease.

\section{Current treatment for an acute episode of diverticulitis}

Specifics around the diagnosis and treatment of acute diverticulitis are based on single papers in most cases; few high-quality randomized trials, systematic reviews, 
or meta-analyses have been published. Despite the lack of evidence from high quality studies, there is some evidence to support approaches to treatment.

Currently, the "blind-pouch", which implicates fecal stasis and bacterial overgrowth, is the most accepted theory explaining the occurrence of diverticular inflammation. ${ }^{5,6}$ Hence, the first stage of treatment for mild symptoms may involve a high-fiber diet, along with antibiotic therapy. ${ }^{1,7,8}$ Meperidine is the preferred option for control of severe abdominal pain, given that morphine causes colonic spasm and may accentuate colonic hypersegmentation with a higher risk of colonic perforation. ${ }^{1,6,9}$

In the vast majority of cases, inflammation in diverticulitis is mild. Patients with uncomplicated diverticulitis are generally treated with a clear liquid diet and antibiotics on an outpatient basis. ${ }^{9}$ A recent retrospective study evaluated how many patients at first episode of acute diverticulitis could be managed as outpatients. ${ }^{10}$ The diagnosis of acute diverticulitis was confirmed by computed tomography (CT), and endpoints included length of stay, need for surgery, percutaneous drainage, and mortality. Patients were considered to have had a minimal hospitalization, defined as survival to discharge without needing a procedure, hospitalization of $\leq 3$ days, and no readmission for diverticulitis within 30 days after discharge. In a cohort of 639 patients, 368 (57.6\%) had minimal hospitalization. Female sex and CT scan findings of free air/fluid were negatively associated with the likelihood of minimal hospitalization. The presence of an abscess $<3 \mathrm{~cm}$ and stranding on CT did not predict the need for a higher level of care. Unfortunately, the authors failed to identify patients likely to need only minimal hospitalization, and only free air/ liquid in a patient admitted for acute diverticulitis indicates a more severe clinical course. ${ }^{10}$

\section{Outpatient treatment of diverticulitis}

The majority of uncomplicated diverticulitis can be safely managed on an outpatient basis. For example, in a retrospective analysis, Etzioni et al found that outpatient treatment was effective for the vast majority (94\%) of patients suffering from acute diverticulitis. ${ }^{11}$ Other than a clear liquid diet, broadspectrum antibiotics are usually given for 7-10 days. Various antibiotics may be used in the treatment of acute diverticulitis in order to ensure complete coverage against Gram-positive, Gram-negative, and aerobic-anaerobic bacterial strains. ${ }^{1,6,8}$ The combination of ciprofloxacin and metronidazole is commonly used for uncomplicated diverticulitis, ${ }^{1,6,8}$ both intravenously and orally, but sometimes these antibiotics may be poorly tolerated by some patients because of their high systemic absorption. Therefore, the American Society of Colon and Rectal Surgeons recommends ampicillin-sulbactam as a good choice in uncomplicated diverticulitis. ${ }^{12}$

As stated, few high-quality randomized trials have been published in this field (see Table 1). One of them, published in 2010, compared the efficacy of short-term therapy (4 days) versus standard therapy (7 days) for uncomplicated sigmoid diverticulitis using ertapenem $1 \mathrm{~g}$ daily. Both patient groups were monitored until discharge and then followed up after 4-6 weeks and 52 months. No significant differences were found between the two groups in terms of the basic data, apart from the mean number of diverticulitis episodes (short-term 1.28 \pm 0.64 versus standard 1.64 $\pm 1.07, P=0.037$ ). The mean hospital stay was 8.8 days, and was significantly lower in the short-term group than in the standard therapy group ( $7.8 \pm 2.8$ days versus $9.7 \pm 3.2$ days; $P=0.002$ ). After 4 days, treatment was successful in $98.0 \%$ of cases and after 7 days in $98.2 \%$ of cases. An overall success rate of $95.1 \%$ (94.0\% versus $96.2 \%$, not statistically significant) was recorded after 1 month. ${ }^{13}$

Another recent prospective study compared the efficacy, safety, and costs of hospital treatment with intravenous antibiotics and outpatient treatment with oral antibiotics. Of 76 patients included in the study, 44 underwent intravenous treatment with metronidazole $500 \mathrm{mg}$ three times daily plus ciprofloxacin $400 \mathrm{mg}$ twice daily (hospital treatment group) and 32 took oral metronidazole $500 \mathrm{mg}$ three times daily

Table I Randomized studies for outpatient treatment of uncomplicated diverticulitis

\begin{tabular}{|c|c|c|c|}
\hline Author & Treatment & End-points & Results \\
\hline Schug-Pass ${ }^{13}$ & Short vs standard treatment & Number of diverticulitis episodes & $P=0.037$ \\
\hline \multirow[t]{2}{*}{ Moya $^{14}$} & Outpatient vs inpatient treatment & I. Obtaining remission & $P=0.86$ \\
\hline & & 2. Saving costs & $P \leq 0.05$ \\
\hline Unlü15 & Outpatient vs inpatient treatment & Rate of complications remission & $P=n s$ \\
\hline \multirow[t]{2}{*}{ Lorente $^{17}$} & Outpatient vs inpatient treatment & I. Diverticulitis recurrence & $P=0.7$ \\
\hline & & 2. Saving costs & $P=0.000 I$ \\
\hline \multirow[t]{2}{*}{ Biondo ${ }^{16}$} & Outpatient vs inpatient treatment & I. Diverticulitis recurrence & $P=0.619$ \\
\hline & & 2. Saving costs & $P=0.001$ \\
\hline
\end{tabular}

Abbreviations: vs, versus; ns, not significant. 
and ciprofloxacin $500 \mathrm{mg}$ twice daily (outpatient group). Outpatient treatment was viable in almost $95 \%$ of those patients suffering from uncomplicated acute diverticulitis, since only two patients $(6 \%)$ required hospital admission after outpatient treatment. Rates of complications and relapse were similar between patients admitted to hospital and treated with intravenous antibiotics and those treated as outpatients with oral antibiotics $(P=0.86)$. Finally, the outpatient approach was able to save approximately $€ 1,600$ per patient $(P<0.05){ }^{14}$

A recent retrospective trial assessed whether outpatient treatment of acute uncomplicated diverticulitis is feasible and safe, and which patients could benefit from outpatient care. Exclusion criteria were recurrent diverticulitis, complicated diverticulitis (Hinchey stages 2-4), and right-sided diverticulitis. Inpatient care was compared with outpatient care. The primary outcomes were admission for outpatient care and the complication rate in both groups. Multivariate analysis was carried out to identify potential factors for inpatient care. Of 627 patients with diverticulitis, 312 consecutive patients were identified with primary uncomplicated sigmoid diverticulitis; 194 patients were treated as inpatients and 118 primarily as outpatients. In the outpatient group, 91.5\% were treated successfully without diverticulitis-related complications or need for hospital admission during a mean follow-up period of 48 months. Despite the patient selection inherent in a retrospective cohort, this study confirms that ambulatory treatment of patients presenting with uncomplicated acute diverticulitis seems feasible and safe, especially in mildly ill and younger patients, thereby avoiding hospital admission. $^{15}$

There are no clear recommendations regarding whether diverticulitis found on colonoscopy should be always treated. A first diagnosis of diverticulitis by colonoscopy is not a rare occurrence. ${ }^{5}$ A reasonable approach is to treat the patient if there are any relevant symptoms or elevated inflammatory markers. If the patient is completely asymptomatic, as sometimes occurs when undergoing a screening colonoscopy for cancer, a reasonable approach could be a short course of treatment followed by strict clinical surveillance.

\section{Quality of life and economic costs of current outpatient treatment for acute diverticulitis}

A very recent trial compared inpatient (group 1) versus outpatient (group 2) management of uncomplicated left colonic diverticulitis and analyzed differences in quality of life and economic costs. The first dose of antibiotic was given intravenously to all patients in the emergency department; group 1 patients were hospitalized whereas group 2 patients were discharged. The primary endpoint was the failure rate of the outpatient treatment protocol and need for hospital admission. Secondary endpoints included a quality of life assessment and evaluation of costs. Of 132 patients randomized, four in group 1 and three in group 2 had treatment failure; this difference was not statistically significant $(P=0.619)$. The overall health care cost per episode was three times lower in group 2, with savings of $€ 1,124.70$ per patient. No difference was observed between the groups in terms of quality of life. ${ }^{16}$

A recent retrospective cohort study compared the impact of outpatient treatment on reduction of health care costs. A total of 136 patients were included, with 90 in the outpatient treatment group (ie, a liquid diet for the first 2 days and oral amoxicillin-clavulanate $1 \mathrm{~g}$ three times daily or associated ciprofloxacin $500 \mathrm{mg}$ two times daily and metronidazole $500 \mathrm{mg}$ three times daily in patients allergic to penicillin for 7 days, combined with oral paracetamol $1 \mathrm{~g}$ three times/daily) and 46 in the hospital treatment group (intravenous cefotaxime $1 \mathrm{~g}$ four times daily and metronidazole $500 \mathrm{mg}$ every 8 hours, or intravenous amoxicillinclavulanate $1 \mathrm{~g}$ three times daily for 7 days). Cost estimates were done using the hospital cost accounting system based on total costs, the sum of all variable costs (direct costs) plus overhead expenses divided by activity (indirect costs). There were no differences in the characteristics of the patients in the two treatment groups. There was also no difference in the treatment failure rate between the groups ( $5.5 \%$ versus $4.3 \%$; $P=0.7)$. The mean total cost per episode was significantly lower in the outpatient treatment group (€882 $€ 462$ versus $€ 2,376 \pm € 830 ; P=0.0001) .{ }^{17}$

\section{Are antibiotics always mandatory in uncomplicated diverticulitis?}

These trials confirm that outpatient treatment is safe and effective in selected patients with uncomplicated acute diverticulitis. Outpatient treatment allows important costs saving to health systems without a negative influence on quality of life in patients with uncomplicated diverticulitis, and reduces health care costs by more than $60 \%$. Unfortunately, the efficacy of antibiotic use in acute diverticulitis, uncomplicated disease included, is not "evidence-based". Two recent randomized studies found that antibiotic treatment was not superior to simple support therapy in terms of obtaining clinical resolution and preventing recurrence of diverticulitis. 
A recent retrospective case-control study was performed in 272 patients with mild colonic diverticulitis admitted to two hospitals with distinctly different treatment regimens concerning antibiotic use. In total, 191 patients were treated without antibiotics and 81 with antibiotics. The two groups were comparable at baseline with respect to age, sex, comorbidity, use of nonsteroidal anti-inflammatory drugs, steroids and aspirin, C-reactive protein levels, and white cell count. In the antibiotic group, there were significantly more patients with a temperature of $38.5^{\circ} \mathrm{C}$ or higher on admission $(8 \%$ versus $19 \% ; P=0.014)$. The treatment failure rate did not differ between the groups ( $4 \%$ versus $6 \% ; P=0.350$ ). The risk of recurrence was higher in the antibiotic group on logistic regression analysis but did not reach statistical significance (odds ratio 2.04; 95\% confidence interval $0.88-4.75$; $P=0.880$ ). The only factor that increased the risk of recurrence was nonsteroidal anti-inflammatory drug use (odds ratio 7.25; 95\% confidence interval 1.22-46.88; $P=0.037){ }^{18}$

A multicenter, randomized trial conducted in Sweden and Iceland recruited 623 patients with CT-confirmed acute uncomplicated left-sided diverticulitis. These patients were randomized to treatment with or without antibiotics (314 versus 309 patients, respectively). Age, sex, body mass index, comorbidities, body temperature, white cell count, and C-reactive protein level on admission were similar in the two groups. Complications, such as perforation or abscess formation, were found in six patients $(1.9 \%)$ who did not receive antibiotics and in three patients $(1.0 \%)$ who received antibiotics $(P=0.302)$. The median hospital stay was 3 days in both groups. The rate of recurrent diverticulitis requiring hospital readmission at one-year follow-up was similar in the two groups $(16 \%, P=0.881){ }^{19}$

These trials show that antibiotic treatment for acute uncomplicated diverticulitis neither accelerates recovery nor prevents complications or recurrence, and that antibiotics should be reserved for treatment of complicated diverticulitis. Why this occurs is unclear, and the use of antibiotics in treating both symptomatic uncomplicated diverticular disease and acute diverticulitis is becoming questionable. Some interesting trials are currently ongoing in order to answer these questions..$^{20,21}$

\section{When outpatient treatment of uncomplicated diverticulitis fails}

Outpatient treatment is effective in most cases, with less than $10 \%$ of patients require readmission to the emergency room for diverticulitis within 60 days of initial evaluation. ${ }^{1,6}$ However, if the patient is unable to take oral therapy, is affected by severe comorbidity, or fails to improve with outpatient therapy, ${ }^{1,6}$ the current guidelines usually recommend that the patient be treated in hospital with intravenous antibiotics. ${ }^{15,16}$ Clinical improvement in patients affected by acute diverticulitis is generally observed within 3-4 days. If patients are admitted to hospital, a 7-10-day course of oral antibiotics is usually given following discharge. . $^{1,6,9,22}$

\section{Unconventional treatments for uncomplicated diverticulitis}

A recent, retrospective, open-label trial established the role of Japanese herbal (Kampo) medicine, (daiobotanpito or Da Huang Mu Dan Tang in Chinese) in the treatment of acute diverticulitis. ${ }^{23}$ Daiobotanpito has been used in traditional Kampo medicine for the treatment of abscesses of the intestine, such as diverticulitis or complications of appendicitis. ${ }^{24}$ In this study, patients received intravenous antibiotics with (group 1) or without (group 2) daiobotanpito. Supplementation with daiobotanpito was significantly better in reducing duration of fever $(P<0.05)$, abdominal pain $(P<0.05)$, and antibiotic administration $(P<0.05)$. A trend toward a shorter hospital stay $(P=0.061)$ and shorter fasting $(P=0.055)$ was found, but this did not reach statistical significance. Although limited by its open-label and retrospective design, this study opens up an interesting debate on the use of traditional herbal medicine as support to standard medical treatment.

\section{Current management of diverticulitis following an acute episode}

Looking at the larger, longer-term studies, we know that the long-term recurrence rate of diverticulitis is up to $20 \%,{ }^{17-19}$ even if a more recent, colonoscopy-based study hypothesized a lower rate of disease occurrence. ${ }^{3}$ Despite these data, there is little evidence to define the optimal approach to management of diverticulitis following an acute episode in order to prevent recurrence.

\section{High-fiber diet}

Once the acute episode has resolved, patients are generally advised to maintain a high-fiber diet in order to optimize their bowel movements. ${ }^{22}$ However, the current literature investigating the role of dietary modification in preventing recurrence of diverticulitis shows conflicting results, ${ }^{25-27}$ and there is not consistent support for recommending a high-fiber diet. However, a high-fiber diet is still commonly recommended to reduce the likelihood of recurrence of diverticulitis despite 
this lack of evidence. ${ }^{9}$ Another interesting point relates to the classical advice to avoid consuming seeds, popcorn, and nuts, which is based on the assumption that such substances could theoretically enter, block, or irritate a diverticulum, resulting in diverticulitis and increased risk of perforation. There is, however, no evidence to date to support this practice. ${ }^{28}$

\section{Antibiotics}

Given the potential involvement of microbial imbalance in the pathogenesis of diverticular disease, ${ }^{1}$ one option to prevent recurrence of diverticulitis may be to use a single broad-spectrum antibiotic covering both Gram-negative and anaerobic bacteria. Rifaximin, a poorly absorbed broad-spectrum oral antibiotic, has been shown to be effective against Gram-positive and Gram-negative aerobic and anaerobic bacteria. ${ }^{1}$ A recent open-label pilot study found that cyclic administration of rifaximin $(800 \mathrm{mg} /$ day for 10 days every month) can effectively improve symptoms and maintain periods of remission following diverticulitis. ${ }^{29}$ However, the results of two recent systematic reviews in assessing the role of rifaximin in preventing recurrence of diverticulitis are negative. ${ }^{30,31}$ The reason why these conflicting results occur is not clear. Varying study quality regarding this specific outcome may be a possible explanation. However, it is likely that the ineffectiveness of rifaximin in preventing recurrence of diverticulitis may be caused by the short-lasting effect of the reduction of fecal bacterial counts during oral treatment with rifaximin. In fact, we know that the colonic bacterial population recovers within 1-2 weeks after the end of treatment. ${ }^{32}$ Thus, a 10-day oral regimen with rifaximin (as assessed in the study by Lanas et $\mathrm{al}^{29}$ ) may control the colonic bacterial population for only 15-20 days. A high colonic bacterial concentration persists therefore for the last 10 days of the month, with a high risk of recurrence of diverticulitis.

\section{Surgery}

Surgery is considered a therapeutic option after attacks of diverticulitis. Current guidelines ${ }^{8,33}$ advise elective resection after two well documented attacks of diverticulitis, depending on the severity of the attack and age and medical fitness of the patient. However, recent data indicate that one quarter of patients experience persistent abdominal symptoms following elective surgery for diverticulitis. ${ }^{34}$ Neither the stage of disease (complicated or uncomplicated) nor the surgical technique (laparotomy or laparoscopy) were significantly related to the occurrence of symptoms. ${ }^{34}$
Nowadays, more recent studies ${ }^{35,36}$ have shown that the natural history of acute diverticulitis is much more benign than thought in the past, and current guidelines no longer support prophylactic surgery for acute diverticulitis, but state that the decision for elective resection in patients with previous acute episodes should be made on a "case-by-case" basis. A more individualized approach taking into account the frequency and severity of attacks and their impact on quality of life should therefore guide the indication for surgery. ${ }^{32}$

\section{5-aminosalicylic acid}

5-Aminosalicylic acid (or mesalamine) is the primary therapy used for induction and maintenance of remission for mild to moderate severe inflammatory bowel disease, in particular ulcerative colitis. As a result of its anti-inflammatory and immunomodulatory properties, a number of studies have recently investigated the influence of mesalamine on the signs and symptoms of diverticular disease. Two recent, randomized, double-blind, placebo-controlled studies found that mesalamine, alone or in combination with probiotics, was effective in controlling abdominal symptoms (mainly pain) and that at a dosage of $3 \mathrm{~g} /$ day it was significantly better than placebo in preventing diverticulitis. ${ }^{37,38}$

The use of mesalamine in preventing recurrence of diverticulitis, however, may be more interesting. Seven double-blind, placebo-controlled studies assessing the role of mesalamine in preventing recurrence of diverticulitis have recently been reported. ${ }^{39-44}$ Unfortunately, most did not find mesalamine to be better than placebo in preventing recurrence of diverticulitis. However, the DIV/04 (Evaluation of Efficacy of Mesalamine in the Long-term Prevention of Diverticulitis Flares) trial found a decrease in the relative risk of recurrence after 24 months in a mesalamine group compared with a placebo group ( $11 \%$ versus $28 \%$, respectively; $P=0.48,95 \%$ confidence interval $0.20-1.15) .{ }^{40}$ On the other hand, mesalamine was found to be significantly better than placebo in reducing abdominal symptoms following acute diverticulitis (Asacol Acute Diverticulitis trial, $P=0.045 ; 36$ DIV/04 trial, $\left.P=0.021^{40}\right)$.

Only one trial conducted in Romania found that mesalamine ( $514.7 \pm 30.5 \mathrm{mg} /$ day) was better than placebo with regard to several endpoints over a 40-month period, ie, reduction of risk of diverticulitis $(P=0.044)$, number of diverticulitis flares $(P=0.001)$, and need for surgery $(P=0.02)$. The relative risk of developing diverticulitis was 2.47 times higher (95\% confidence interval $1.38-4.43)$ in the placebo group than in the mesalamine group. ${ }^{44}$ Although these results seem disappointing, a caution in commenting on these data 
is still needed because a lot of bias affected most of these trials (heterogeneity in the population enrolled, different types of mesalamine discharged through the colon, heterogeneity in endpoints assessed). These important points need to be addressed by future studies.

\section{Probiotics}

By definition, probiotics are living microorganisms that can alter the host microflora and exert specific health benefits without increasing the risk of antibiotic resistance. ${ }^{45}$ In recent years, some open-label studies have evaluated the role of probiotics in the treatment of diverticulitis-related complications or in preventing recurrence of diverticulitis. More than 20 years ago, using Lactobacillus sp. following rifaximin in 79 patients with colonic stenosis post-diverticulitis, Giaccari et al found that the vast majority remained symptom-free for a period of one year. ${ }^{46}$ No repeated episodes of diverticulitis occurred during the follow-up period, and stenoses improved or remained stable in half of their patients. ${ }^{46}$ Although this observational study lacked a placebo arm, it is significant because it is the first study suggesting the role of probiotics in preventing occurrence/recurrence of the inflammatory complications of diverticular disease.

A more recent study investigated the combination of balsalazide, a 5-aminosalicylic acid prodrug, with the high-potency, probiotic mixture VSL\#3 ${ }^{\circledR}$ (composed of eight different bacterial strains, ie, Lactobacillus casei, Lactobacillus plantarum, Lactobacillus acidophilus, Lactobacillus delbrueckii subsp. bulgaricus, Bifidobacterium longum, Bifidobacterium breve, Bifidobacterium infantis, and Streptococcus salivarius subsp. thermophilus) in preventing recurrence of diverticulitis. The authors found that the combination of balsalazide and VSL\#3 was better than VSL\#3 alone in preventing recurrence of diverticulitis, even if their results did not reach statistical significance $(73.33 \%$ versus $60 \%, P<0.1) .^{47}$

Finally, an interesting approach in preventing diverticulitis may be the use of bacterial lysate. The intestine is well known to be the largest human lymphoepithelial organ, and on a daily basis produces more antibodies, mainly secretory immunoglobulin A, than do all other lymphoid tissues. Ten years ago, Dughera et al assessed the efficacy of an oral highly-purified, polymicrobial lysate immunostimulant (containing $80 \times 10^{9}$ Escherichia coli strains 01, 02, 055, and 0111 , and $1 \times 10^{9}$ Proteus vulgaris) in the prevention of recurrent attacks of diverticulitis and in improvement of symptoms. Eighty-three consecutive patients suffering from recurrent symptomatic acute diverticulitis and with at least two attacks in the previous year were randomly assigned to receive an oral polybacterial lysate suspension ( $5 \mathrm{~mL}$ twice daily for 2 weeks every month, group A) or to a no-treatment clinical follow-up as controls group B for a

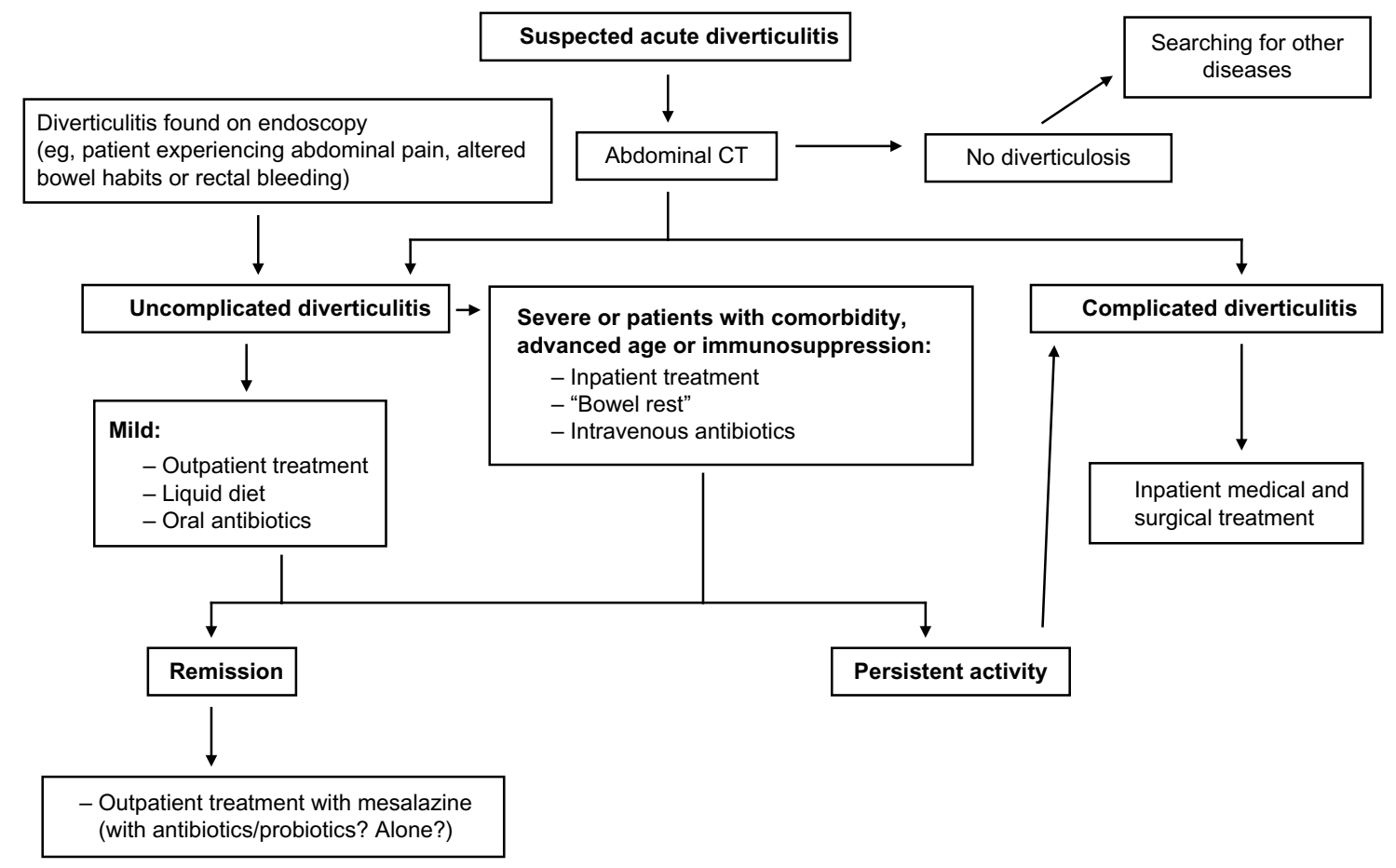

Figure I Proposed algorithm for managing uncomplicated diverticulitis. Abbreviation: $\mathrm{CT}$, computed tomography. 
3-month follow-up period. Statistically significant differences in the sums of the scores between group A and group B were recorded after 1 month $(P<0.05)$ and 3 months $(P<0.01)$ of treatment with the oral polybacterial lysate suspension. ${ }^{48}$ The use of probiotics in preventing recurrence of diverticulitis is therefore intriguing. However, current data are too preliminary to draw any conclusions.

\section{Nutritional treatment}

A recent double-blind, randomized, placebo-controlled study assessed the role of butyrate in preventing recurrence of diverticulitis. ${ }^{49}$ Butyrate is a short-chain fatty acid previously shown to provide symptomatic relief in patients suffering from various colonic diseases, ranging from diarrhea to inflammatory bowel disease, and suggestive of a preventive role in carcinogenesis of colonocytes. ${ }^{50-52}$ Seventy-three patients with at least one episode of diverticulitis not earlier than 1 year before the study were randomized to treatment with microencapsulated sodium butyrate $400 \mathrm{mg}$ daily or placebo. After 12 months, clinical symptoms of diverticulitis occurred in two (6.67\%) and seven (31.8\%) patients in the active and placebo groups, respectively $(P=0.0425) .{ }^{49}$ Although limited by bias, this study supports the hypothesis that a therapy able to influence colonocyte metabolism, reinforcing the colonic mucosal barrier leading to decreased inflammation of the mucosa and increasing the cell regeneration rate with healing of the mucosa is another interesting option in preventing relapses of diverticulitis.

\section{Conclusion}

The medical treatment of uncomplicated diverticulitis is changing (see Figure 1). Although antibiotics seem to remain the mainstay of treatment in the acute phase, treatment on an outpatient basis is now considered to be the optimal therapeutic approach in the vast majority of these patients. This is because outpatient treatment seems to be safe and effective, allowing important cost-savings to health care systems. Further well designed and controlled studies are needed in order to assess whether antibiotics are mandatory or not in the outpatient management of uncomplicated diverticulitis.

\section{Author contributions}

AT made substantial contributions to the conception and design, acquisition of data, and analysis and interpretation of data, as well as drafting the paper and revising it critically for important intellectual content. He approved the final version for publication, and agrees to be accountable for all aspects of the work in ensuring that questions related to the accuracy or integrity of any part of the work are appropriately investigated and resolved.

\section{Disclosure}

The author reports no conflicts of interest in this work.

\section{References}

1. Tursi A, Papagrigoriadis S. Review article: the current and evolving treatment of colonic diverticular disease. Aliment Pharmacol Ther. 2009;30(6):532-546.

2. Weizman AV, Nguyen GC. Diverticular disease: epidemiology and management. Can J Gastroenterol. 2011;25(7):385-389.

3. Shahedi K, Fuller G, Bolus R, et al. Long-term risk of acute diverticulitis among patients with incidental diverticulosis found during colonoscopy. Clin Gastroenterol Hepatol. 2013;11(12):1609-1613.

4. Etzioni DA, Mack TM, Beart RW Jr, Kaiser AM. Diverticulitis in the United States: 1998-2005: changing patterns of disease and treatment. Ann Surg. 2009;249(2):210-217.

5. Tursi A. Advances in the management of colonic diverticulitis. CMAJ. 2012;184(13):1470-1476.

6. Tursi A. Diverticular disease: a therapeutic overview. World $J$ Gastrointest Pharmacol Ther. 2010;1(1):27-35.

7. Lahat A, Yanai H, Menachem Y, Avidan B, Bar-Meir S. The feasibility and risk of early colonoscopy in acute diverticulitis: a prospective controlled study. Endoscopy. 2007;39(6):521-524.

8. Rafferty J, Shellito P, Hyman NH, et al. Practice parameters for sigmoid diverticulitis. Dis Colon Rectum. 2006;49(7):939-944.

9. World Gastroenterology Organisation Practice Guidelines 2007. Diverticular disease. Available from: http://www.worldgastroenterology. org/assets/downloads/en/pdf/guidelines/07_diverticular_disease.pdf. Accessed February 27, 2014.

10. Abbas MA, Cannom RR, Chiu VY, et al. Triage of patients with acute diverticulitis: are some inpatients candidates for outpatient treatment? Colorectal Dis. 2013;15(4):451-457.

11. Etzioni DA, Chiu VY, Cannom RR, Burchette RJ, Haigh PI, Abbas MA. Outpatient treatment of acute diverticulitis: rates and predictors of failure. Dis Colon Rectum. 2010;53(6):861-856.

12. Schechter S, Mulvey J, Eisenstat TE. Management of uncomplicated acute diverticulitis: results of a survey. Dis Colon Rectum. 1999;42(4): 470-475.

13. Schug-Pass C, Geers P, Hügel O, Lippert H, Köckerling F. Prospective randomized trial comparing short-term antibiotic therapy versus standard therapy for acute uncomplicated sigmoid diverticulitis. Int $J$ Colorectal Dis. 2010;25(6):751-759.

14. Moya P, Arroyo A, Pérez-Legaz J, et al. Applicability, safety and efficiency of outpatient treatment in uncomplicated diverticulitis. Tech Coloproctol. 2012;16(4):301-307.

15. Unlü C, Gunadi PM, Gerhards MF, Boermeester MA, Vrouenraets BC. Outpatient treatment for acute uncomplicated diverticulitis. Eur $J$ Gastroenterol Hepatol. 2013;25(9):1038-1043.

16. Biondo S, Golda T, Kreisler E, et al. Outpatients versus hospitalisation management for uncomplicated diverticulitis: a prospective, multicenter randomised clinical trial (DIVER Trial). Ann Surg. 2014;259(1): $38-44$.

17. Lorente L, Cots F, Alonso S, et al. Outpatient treatment of uncomplicated acute diverticulitis: impact on healthcare costs. Cir Esp. 2013;91(8):504-509.

18. de Korte N, Kuyvenhoven JP, van der Peet DL, et al. Mild colonic diverticulitis can be treated without antibiotics. A case-control study. Colorectal Dis. 2012;14(3):325-330.

19. Chabok A, Påhlman L, Hjern F, et al. Randomized clinical trial of antibiotics in acute uncomplicated diverticulitis. Br J Surg. 2012;99(4): 532-539. 
20. Unlü C, de Korte N, Daniels L, et al. Multicenter randomized clinical trial investigating the cost-effectiveness of treatment strategies with or without antibiotics for uncomplicated acute diverticulitis (DIABOLO trial). BMC Surg. 2010;10:23.

21. ClinicalTrials.gov. Rifamycin SV-MMX ${ }^{\circledR} 400 \mathrm{mg}$ b.i.d. versus. Rifamycin SV-MMX ${ }^{\circledR} 600 \mathrm{mg}$ t.i.d. versus. placebo in acute uncomplicated diverticulitis. Avalilable from: http://clinicaltrials.gov/ ct2/show/NCT01847664. Accessed February 27, 2014.

22. Stollman NH, Raskin JB. Diagnosis and management of diverticular disease of the colon in adults. Ad Hoc Practice Parameters Committee of the American College of Gastroenterology. Am J Gastroenterol. 1999;94(11):3110-3121.

23. Ogawa K, Nishijima K, Futagami F, Nakamura T, Nishimura G. Effectiveness of traditional Japanese herbal (Kampo) medicine, daiobotampito, in combination with antibiotic therapy in the treatment of acute diverticulitis: a preliminary study. Evid Based Complement Alternat Med. 2013;2013:305414.

24. Scheid V, Bensky D, Ellis A, Barolet R. Chinese Herbal Medicine Formulas and Strategies. 2nd ed. Seattle, WA, USA: Eastland Press; 2009.

25. Crowe FL, Appleby PN, Allen NE, Key TJ. Diet and risk of diverticular disease in Oxford cohort of European Prospective Investigation into Cancer and Nutrition (EPIC): prospective study of British vegetarians and non-vegetarians. BMJ. 2011;343:d4131.

26. Peery AF, Barrett PR, Park D, et al. A high-fiber diet does not protect against asymptomatic diverticulosis. Gastroenterology. 2012;142(2): 266-272.

27. Unlü C, Daniels L, Vrouenraets BC, Boermeester MA. A systematic review of high-fibre dietary therapy in diverticular disease. Int $\mathrm{J} \mathrm{Col}$ orectal Dis. 2012;27(4):419-427.

28. Strate LL, Liu YL, Syngal S, Aldoori WH, Giovannucci EL. Nut, corn, and popcorn consumption and the incidence of diverticular disease. JAMA. 2008;300(8):907-914.

29. Lanas A, Ponce J, Bignamini A, Mearin F. One year intermittent rifaximin plus fibre supplementation versus. Fibre supplementation alone to prevent recurrence of diverticulitis: a proof-of-concept study. Dig Liver Dis. 2013;45(2):104-109.

30. Zullo A, Hassan C, Maconi G, et al. Cyclic antibiotic therapy for diverticular disease: a critical reappraisal. $J$ Gastrointest Liver Dis. 2010;19(3):295-302.

31. Maconi G, Barbara G, Bosetti C, Cuomo R, Annibale B. Treatment of diverticular disease of the colon and prevention of acute diverticulitis: a systematic review. Dis Colon Rectum. 2011;54(10):1-12.

32. Scarpignato C, Pelosini I. Experimental and clinical pharmacology of rifaximin, a gastrointestinal selective antibiotic. Digestion. 2006; 73 Suppl 1:15-38.

33. Stocchi L. Current indications and role of surgery in the management of sigmoid diverticulitis. World J Gastroenterol. 2010;16(7): 804-817.

34. Egger B, Peter MK, Candinas D. Persistent symptoms after elective sigmoid resection for diverticulitis. Dis Colon Rectum. 2008;51(7): 1044-1048.

35. Eglinton T, Nguyen T, Raniga S, et al. Patterns of recurrence in patients with acute diverticulitis. Br J Surg. 2010;97(6):952-957.

36. Binda GA, Arezzo A, Serventi A, et al. Multicentre observational study of the natural history of left-sided acute diverticulitis. Br J Surg. 2012;99(2):276-285.

Drug, Healthcare and Patient Safety

\section{Publish your work in this journal}

Drug, Healthcare and Patient Safety is an international, peer-reviewed open-access journal exploring patient safety issues in the healthcare continuum from diagnostic and screening interventions through to treatment, drug therapy and surgery. The journal is characterized by the rapid reporting of reviews, original research, clinical, epidemiological and
37. Kruis W, Meier E, Schumacher M, et al. Randomised clinical trial: mesalazine (Salofalk granules) for uncomplicated diverticular disease of the colon - a placebo-controlled study. Aliment Pharmacol Ther. 2013;37(7):680-690.

38. Tursi A, Brandimarte G, Elisei W, et al. Randomised clinical trial: mesalazine and/or probiotics in maintaining remission of symptomatic uncomplicated diverticular disease - a double-blind, randomised, placebo-controlled study. Aliment Pharmacol Ther. 2013;38(7): 741-751.

39. Stollman N, Magowan S, Shanahan F, et al. A randomized controlled study of mesalamine after acute diverticulitis: results of the DIVA trial. J Clin Gastroenterol. 2013;47(7):621-629.

40. Parente F, Bargiggia S, Prada A, et al. Intermittent treatment with mesalazine in the prevention of recurrence of diverticulitis: a randomised multicentre pilot double-blind placebo-controlled study of 24-month duration. Int $J$ Colorectal Dis. 2013;28(10):1423-1431.

41. Prevention of Recurrence of Diverticulitis (PREVENT 1) (NCT00545740). Available from: http://www.clinicaltrials.gov/ct2/ show/NCT00545740. Accessed February 27, 2014.

42. Prevention of Recurrence of Diverticulitis (PREVENT 2) (NCT00545103). Available from: http://www.clinicaltrials.gov/ct2/ show/NCT00545103. Accessed February 27, 2014.

43. Kruis W, Eisenbach T, Löhr H, et al. Double-blind, randomized, placebo-controlled, multicenter trial of mesalamine for the prevention of recurrence of diverticulitis. Gastroenterology. 2013;144 Suppl 1: S139.

44. Gaman A, Teodorescu R, Georhescu EF, Abagiu MT. Prophylactic effects of mesalamine in diverticular disease. Abstract 13 presented at the Falk Symposium 178, September 2-3, 2011, Cologne, Germany.

45. Isolauri E, Sütas Y, Kankaanpää P, Arvilommi H, Salminen S. Probiotics: effects on immunity. Am J Clin Nutr. 2001;73(Suppl 2):444S-450S.

46. Giaccari S, Tronci S, Falconieri M, Ferrieri A. Long-term treatment with rifaximin and lactobacilli in post-diverticulitic stenoses of the colon. Eur Rev Med Pharmacol Sci. 1993;15(1):29-34.

47. Tursi A, Brandimarte G, Giorgetti GM, Elisei W, Aiello F. Balsalazide and/or high-potency probiotic mixture (VSL\#3) in maintaining remission after attack of acute, uncomplicated diverticulitis of the colon. Int J Colorectal Dis. 2007;22(9):1103-1108.

48. Dughera L, Serra AM, Battaglia E, Tibaudi D, Navino M, Emanuelli G. Acute recurrent diverticulitis is prevented by oral administration of a polybacterial lysate suspension. Minerva Gastroenterol Dietol. 2004;50(2):149-153.

49. Krokowicz L, Stojcev Z, Kaczmarek BF, et al. Microencapsulated sodium butyrate administered to patients with diverticulosis decreases incidence of diverticulitis - a prospective randomized study. Int $J$ Colorectal Dis. 2014;29(3):387-393.

50. Banasiewicz T, Krokowicz L, Stojcev Z, et al. Microencapsulated sodium butyrate reduces the frequency of abdominal pain in patients with irritable bowel syndrome. Colorectal Dis. 2013;15(2):204-209.

51. Cordel S, Dupas B, Douillard JY, Meflah K. Interleukin-2/sodium butyrate treatment cures rats bearing liver tumors after acquired 5-fluorouracil resistance. Int J Cancer. 1998;78(6):735-739.

52. Vernia $P$, Annese V, Bresci G, d'Albasio G, D'Incà R, Giaccari S. Topical butyrate improves efficacy of 5-ASA in refractory distal ulcerative colitis: results of a multicentre trial. Eur J Clin Investig. 2003;33(3):244-248

post-marketing surveillance studies, risk management, health literacy and educational programs across all areas of healthcare delivery. The manuscript management system is completely online and includes a very quick and fair peer-review system. Visit http://www.dovepress.com/ testimonials.php to read real quotes from published authors. 